\title{
Erratum: Accurate electronic band gaps of two-dimensional materials from the local modified Becke-Johnson potential [Phys. Rev. B 101, 245163 (2020)]
}

Tomáš Rauch, Miguel A. L. Marques, and Silvana Botti

(Received 14 September 2020; published 24 September 2020)

DOI: 10.1103/PhysRevB.102.119902

In the paper, we tested the accuracy of the local modified Becke-Johnson (LMBJ) exchange-correlation potential for the prediction of band gaps of two-dimensional (2D) materials.

We first optimized the $\sigma$ and $r_{s}^{\text {th }}$ parameters of the potential by calculating the electronic band gaps of for 22 selected semiconductors. Some of our original calculations were not fully converged with respect to the k-point density and the vacuum

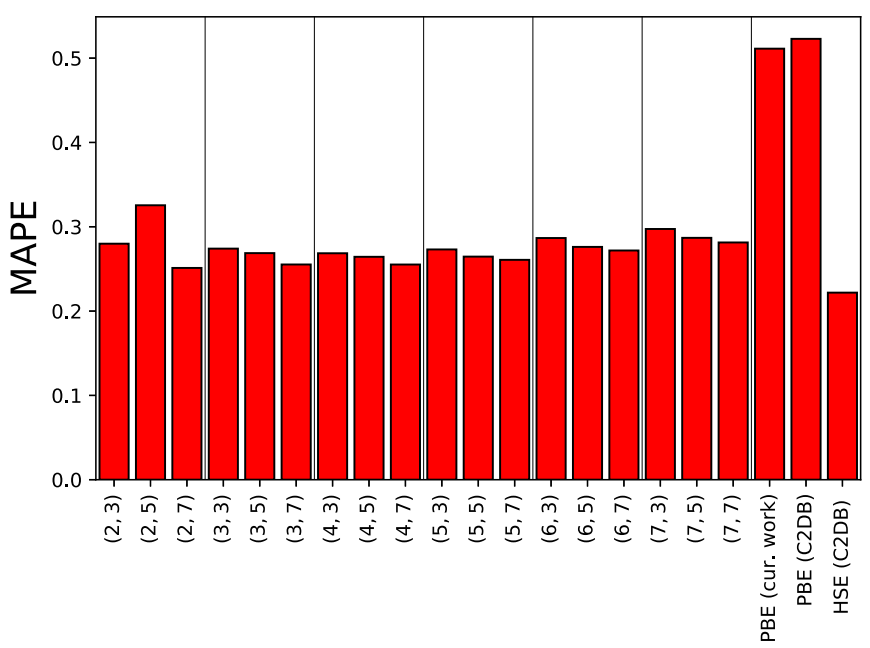

FIG. 1. Mean absolute percentage error (MAPE) for different values of $\left(\sigma, r_{s}^{\text {th }}\right)$ parameters compared with Perdew-Burke-Ernzerhof (PBE) and Heyd-Scuseria-Ernzerhof (HSE06) values.

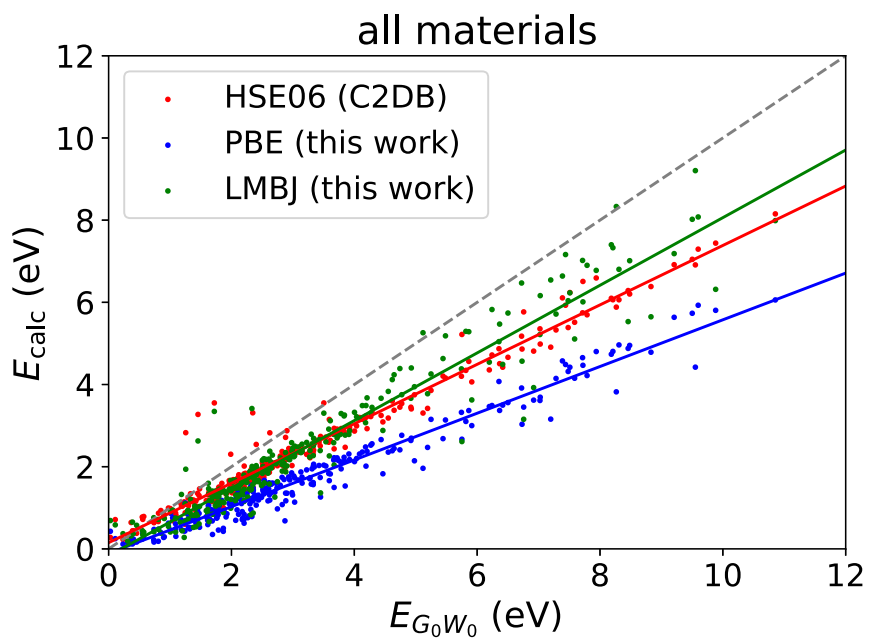

FIG. 2. Calculated band gaps as a function of $G_{0} W_{0}(\mathrm{C} 2 \mathrm{DB})$ band gaps. Full lines are linear fits $(y=a x+b)$ to the respective data with $a$ and $b$ given in Table I. 
TABLE I. Statistical measures for calculations of band gaps of 2D materials with HSE06 (from C2DB), PBE, and LMBJ potentials for the data sets "all materials," $s p$ materials, and $d$ materials. All errors are calculated with respect to the $G_{0} W_{0}$ band gaps from C2DB. The statistical measures are, in order, the number of false metals, mean error (ME, in eV), MAE, in eV, mean percentage error (MPE), MAPE, standard deviation $(\sigma$, in $\mathrm{eV})$, interquartile range (IQR, in $\mathrm{eV})$, and linear fit $(y=a x+b)$ coefficients.

\begin{tabular}{lccccccccrrr}
\hline \hline Set & XC & No. & False metal & ME & MAE & MPE & MAPE & $\sigma$ & IQR & $a$ & $b$ \\
\hline All & HSE06 (C2DB) & 276 & 0 & 0.73 & 0.79 & 0.14 & 0.30 & 0.68 & 0.59 & 0.72 & 0.15 \\
All & PBE & 276 & 0 & 1.48 & 1.48 & 0.42 & 0.53 & 0.96 & 0.93 & 0.57 & -0.11 \\
All & LMBJ & 276 & 0 & 0.73 & 0.78 & 0.14 & 0.38 & 0.62 & 0.46 & 0.82 & -0.17 \\
$s p$ & HSE06 (C2DB) & 161 & 0 & 0.90 & 0.90 & 0.25 & 0.25 & 0.60 & 0.56 & 0.73 & 0.05 \\
$s p$ & PBE & 161 & 0 & 1.58 & 1.58 & 0.46 & 0.46 & 0.94 & 0.85 & 0.58 & -0.10 \\
$s p$ & LMBJ & 161 & 0 & 0.80 & 0.82 & 0.22 & 0.24 & 0.62 & 0.53 & 0.77 & 0.003 \\
$d$ & HSE06(C2DB) & 115 & 0 & 0.48 & 0.63 & -0.01 & 0.36 & 0.70 & 0.67 & 0.74 & 0.23 \\
$d$ & PBE & 115 & 0 & 1.33 & 1.34 & 0.37 & 0.64 & 0.98 & 1.10 & 0.55 & -0.11 \\
$d$ & LMBJ & 115 & 0 & 0.64 & 0.72 & 0.04 & 0.57 & 0.61 & 0.51 & 0.90 & -0.37 \\
\hline \hline
\end{tabular}
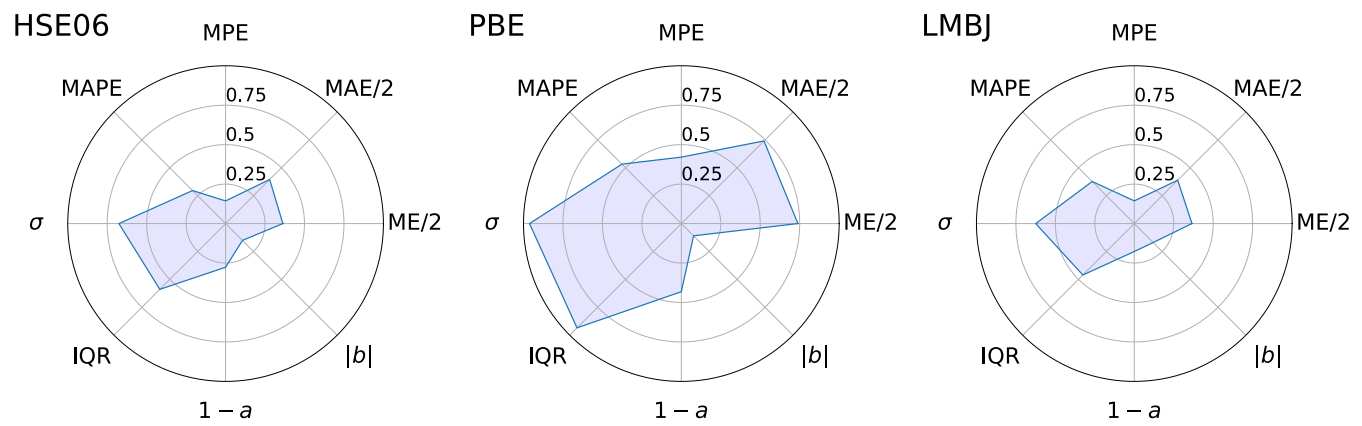

FIG. 3. Radar charts of the statistical quantities in Table I for HSE06 (C2DB), PBE, and LMBJ.
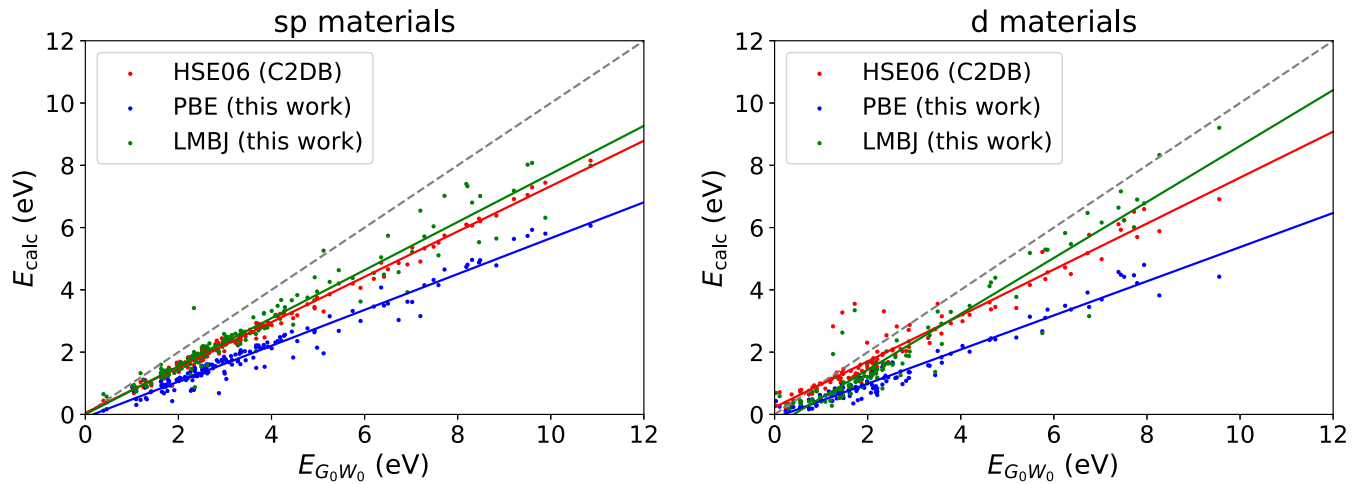

FIG. 4. Calculated band gaps as a function of $G_{0} W_{0}$ (C2DB) band gaps for the data sets $s p$ materials (left) and $d$ materials (right). Full lines are linear fits $(y=a x+b)$ to the respective data with $a$ and $b$ given in the main text.
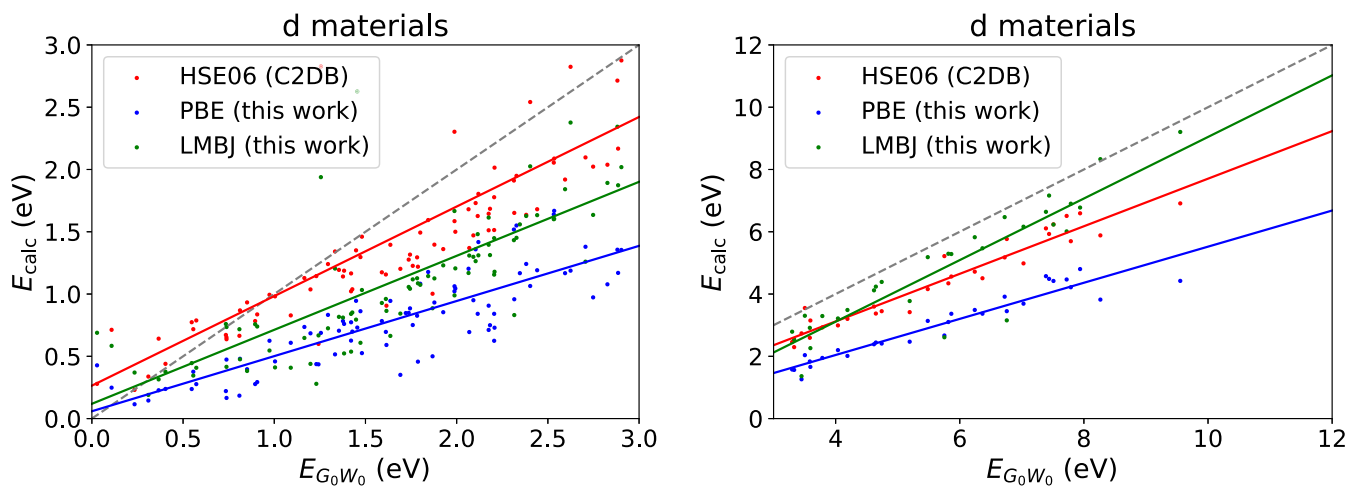

FIG. 5. Calculated band gaps as a function of $G_{0} W_{0}(\mathrm{C} 2 \mathrm{DB})$ band gaps for the data sets $d$ materials with $E_{G_{0} W_{0}} \in(0,3) \mathrm{eV}(\mathrm{left})$ and $d$ materials with $E_{G_{0} W_{0}} \in(3,12) \mathrm{eV}$ (right). Full lines are linear fits $(y=a x+b)$ to the respective data with $a$ and $b$ given in the main text. 
thickness (the geometries were originally obtained from the computational 2D materials database (C2DB) database [1]). We repeated all calculations with a higher k-point density of $14.0 / \AA^{-1}$ and two periodic $2 \mathrm{D}$ replicas were separated by $30 \AA$ of vacuum (45 $\AA$ for BN). The new results are summarized in Fig. 1 (cf. Fig. 2 of the Supplemental Material of the original paper). We observe a small change in individual calculated band-gap values, but the MAPE with respect to the $G_{0} W_{0}$ results remains mostly below 30\%. In the refined calculations, there are no false metal predictions and the setting with the lowest MAPE (25.1\%) is $\sigma=3.78$ bohrs $=2 \AA$ and $r_{s}^{\text {th }}=7$ bohrs (in contrast to $\sigma=4 \AA$ and $r_{s}^{\text {th }}=5$ bohrs of the original paper).

In a second step, we evaluated the quality of the optimized LMBJ potential by calculating the band gaps of 276 2D materials. Having new optimal parameter values, we repeated also these calculations with the improved settings as in the first step. We summarize our results in Table I (cf. Table III of the original paper) and Figs. 2 and 3 (cf. Figs. 2 and 3 of the original paper). New results for individual "sp materials" and " $d$ materials" subsets are presented in Figs. 4 and 5 (cf. Figs. 3 and 4 of the Supplemental Material of the original paper).

Note that there are only very small differences in comparison with the results of the original paper. In particular, the MAPE of the optimized LMBJ potential (38\%) remains unchanged. Therefore, the general results of the original paper stay valid.

We conclude that the performance of the LMBJ potential remains close to that of the HSE06 hybrid functional [2,3].

[1] S. Haastrup, M. Strange, M. Pandey, T. Deilmann, P. S. Schmidt, N. F. Hinsche, M. N. Gjerding, D. Torelli, P. M. Larsen, A. C. Riis-Jensen, J. Gath, K. W. Jacobsen, J. J. Mortensen, T. Olsen, and K. S. Thygesen, 2D Mater. 5, 042002 (2018).
[2] J. Heyd, G. E. Scuseria, and M. Ernzerhof, J. Chem. Phys. 118, 8207 (2003).

[3] J. Heyd, G. E. Scuseria, and M. Ernzerhof, J. Chem. Phys. 124, 219906 (2006). 